\title{
Regulation of the insulin-Akt signaling pathway and glycolysis during dehydration stress in the African clawed frog Xenopus laevis
}

\begin{tabular}{|r|l|}
\hline Journal: & Biochemistry and Cell Biology \\
\hline Manuscript ID & bcb-2017-0117 \\
\hline Manuscript Type: & Article \\
\hline Date Submitted by the Author: & $22-$ May-2017 \\
\hline $\begin{array}{r}\text { Complete List of Authors: } \\
\text { Is the invited manuscript for } \\
\text { consideration in a Special } \\
\text { Issue? : }\end{array}$ & $\begin{array}{l}\text { Cessier, Shannon N.; Harvard Medical School, BioMEMS Resource Center \& } \\
\text { Storey, Kenneth B.; Carleton University, Biology }\end{array}$ \\
\hline Keyword: & Desiccation, Metabolic depression, mTOR, HIF-1, Estivation \\
\hline
\end{tabular}




\section{Regulation of the insulin-Akt signaling pathway and glycolysis during dehydration stress in the African clawed frog Xenopus laevis}

Cheng-Wei $\mathrm{Wu}^{1,2}$, Shannon N. Tessier ${ }^{1,3}$, and Kenneth B. Storey ${ }^{1 *}$

${ }^{1}$ Institute of Biochemistry and Department of Biology, Carleton University, 1125 Colonel By

Drive, Ottawa, K1S 5B6, Canada

${ }^{2}$ Current Address: Department of Biology and Genetics Institute, University of Florida, Gainesville, FL 32611, USA

${ }^{3}$ Current Address: BioMEMS Resource Center \& Center for Engineering in Medicine, Massachusetts General Hospital \& Harvard Medical School, 114 16th Street, Charlestown, MA, USA, 02129

Total number of figures: 4

Running title: Regulation of insulin signaling and glycolysis during estivation

\section{*Correspondence to:}

Kenneth B. Storey

Institute of Biochemistry and Department of Biology, Carleton University, 1125 Colonel By Drive, Ottawa, ON, K1S 5B6

Tel: (613) 520-3678

E-mail: kenneth.storey@carleton.ca 


\begin{abstract}
Estivation is an adaptive stress response utilized by some amphibians during periods of drought in the summer season. In this study, we examine the regulation of the insulin signaling cascade and glycolysis pathway in the African clawed frog Xenopus laevis during dehydration stress induced state of estivation. We show that in the brain and heart of $X$. laevis, dehydration reduces the phosphorylation of the insulin growth factor-1 receptor (IGF-1R), and this is followed by similar reductions in the phosphorylation of the Akt and mechanistic target of rapamycin (mTOR) kinase. Interestingly, phosphorylation levels of IGF-1R and mTOR were not affected in the kidney, and phosphorylation levels of P70S6K and ribosomal S6 protein were elevated during dehydration stress. Animals under estivation are also susceptible to periods of hypoxia, suggesting that glycolysis may also be affected. We observed that protein levels of many glycolytic enzymes remained unchanged during dehydration, however, the hypoxia response factor-1 alpha (HIF-1 $\alpha)$ protein was elevated by $>2$-fold in the heart during dehydration. Overall, we provide evidence that show the insulin signaling pathway in $X$. laevis is regulated in a tissue specific manner during dehydration stress, and suggest an important role for this signaling cascade in mediating the estivation response.
\end{abstract}

Key Words: Desiccation; Metabolic depression; mTOR; HIF-1; Estivation 


\section{Introduction}

The African clawed frog Xenopus laevis is commonly used as a model organism to study vertebrate developmental biology and embryology; the Xenopus frogs are an attractive research model as the regulatory mechanisms for many of its biological processes are conserved in human (Karpinka et al. 2015). In its natural aquatic habitat, Xenopus frogs are also an intriguing model to study the physiology of estivation and dehydration stress (Wu et al. 2013). In their native environment of Sub-Saharan Africa, Xenopus frogs are challenged with seasonal drought that forces the animals to adapt to an arid surrounding with little food or water. To defend against this seasonal fluctuation in its aquatic environment, frogs like Xenopus laevis burrow into the soil and enter a state of estivation, which is a prolonged period of inactivity and dormancy (Romspert 1976). In the Xenopus, this adaptive stress response is characterized by dehydration and water loss of the frog by up to $35 \%$ of its body weight, and is accompanied by increases in osmolytes that include ammonia ( 2-3-folds), plasma urea (15-20-folds) and glucose ( 2 -folds) in an effort to increase water retention and to promote moisture uptake from the surrounding soil (Malik and Storey 2009, Storey and Storey 2012). In addition to the water loss and hyperosmotic stress, the arid environment also restricts the food availability for $X$. laevis; as such, $X$. laevis must also tolerate prolonged periods of starvation, which would require significant reorganization of their energy metabolism to survive.

In other animals that experience similar types of environmental constraints (i.e. cold weather induces hibernation in ground squirrels; sub-zero temperature induces freeze tolerance in wood frogs; and oxygen deprivation induces anoxia tolerance in turtles) (Krivoruchko and Storey 2010, Storey 2010, Storey and Storey 2017), entry into a state of metabolic depression has been documented as a strategy to maximize survival during periods of inhabitable conditions. In 
amphibian species such as the spadefoot toad and the African bullfrog, deprivation of food and water has been shown to reduce animal's metabolic rate, suggesting that metabolic depression is also a common stress response utilized by animals during estivation (Loveridge and Withers 1981, Seymour 1973). The establishment of a hypometabolic state would in principle allow the animal to survive prolonged periods of food and water deprivation due to a lowered metabolic demand, and previous studies have shown that $X$. laevis are able endure starvation for up to 12 month without fatality, suggesting that these animals may also possess adaptive capabilities to undergo metabolic depression (Merkle and Hanke 1988). While mechanisms that regulate metabolic depression are well characterized in mammalian models during hibernation, less are known regarding the molecular and cellular processes that are altered in Xenopus frogs during dehydration induced estivation.

One of the major signaling transduction pathway that regulate cellular metabolism is the insulin signaling cascade; in response to extracellular stimuli (i.e. insulin, growth factors, cytokines), the insulin signaling pathway regulate a complex network of cellular processes that include growth and proliferation, carbohydrate and lipid metabolism, along with apoptosis and autophagy (Taniguchi et al. 2006). The insulin signaling pathway is highly conserved from yeast to human, and has been shown to play an integral role in regulating environmental stress responses from dauer and diapause formation in nematodes to hibernation in ground squirrels (Kimura et al. 1997, Wu and Storey 2016). While there are multiple divergent nodes within this signaling network, the core insulin pathway operates through the Akt/PKB (protein kinase B) protein, which is a serine/threonine kinase that can phosphorylate and regulate up to 100 different protein substrates involved in a variety of cellular functions (Carracedo and Pandolfi 2008, Manning and Cantley 2007). Previous studies in animals that undergo environmental stress 
induced metabolic depression has shown that the Akt kinase is inhibited in the ground squirrels during hibernation (Abnous et al. 2008, Cai et al. 2004, McMullen and Hallenbeck 2010, Wu and Storey 2012), and in the wood frogs during winter freezing (Zhang and Storey 2013). It has been proposed that inhibiting the Akt kinase during periods of metabolic stress functions to limit unnecessary cellular growth, and this is partially achieved by impeding the activation of the Akt downstream substrate mTOR (mechanistic target of rapamycin), which is a kinase that positively promotes protein synthesis via activating proteins within the translation machinery (Laplante and Sabatini 2009). More recently, studies have also implicated a role for the mTOR pathway in promoting regulation of glycolysis (Weichhart et al. 2015), supporting a role for the insulin signaling axis in the control of metabolic homeostasis through a wide range of cellular processes.

In this study, we set out to understanding how the insulin signal transduction pathway and glycolysis is regulated in the brain, heart, and kidney of $X$. laevis during dehydration stress. The heart and the brain tissues are of particular interest as they are sensitive to fluctuations in oxygen and blood flow of the animal, and during dehydration stress, amphibians have been reported to experience hypoxia caused by impeded tissue perfusion from elevated blood viscosity (Hillman 1987, 1988). The kidney is also a tissue of interest during dehydration stress, as it is involved in the osmoregulation of the animal, and X. laevis have been reported to show significant increase in levels of several plasma osmolytes including urea during dehydration stress (Malik and Storey 2009, Peña-Villalobos et al. 2016). Here, we provide biochemical data that show key proteins within the insulin signaling axis is suppressed in a tissue specific manner during dehydration stress in $X$. laevis, and implicate a role for this signaling cascade in contributing to metabolic depression during amphibian estivation. 


\section{Materials and Methods}

\section{Animal preparation}

Adult Xenopus laevis were obtained from the Department of Zoology at the University of Toronto, animals were kept in tanks containing dechloraminated water maintained at $22^{\circ} \mathrm{C}$ and fed three times a week (CU Adult Frog diet, PMI Nutrition International). This condition was used to sample control animals. Procedures used to induce dehydration stress were previously described in detail (Malik and Storey 2009), animals procedures used in this study received approval from the Carleton University Animal Care Committee. For medium dehydration stress, $X$. laevis were placed in a plastic bucket and allowed to air dry over time, with $\sim 16-19 \%$ of water loss observed before the animals were sacrificed (Malik and Storey 2009). For high dehydration stress, water loss in $X$. laevis was induced by placing the frogs in a dry plastic bucket that contained silica gel desiccant at the bottom. A perforated divider was placed between the frog and the desiccant to avoid direct contact, with $\sim 26-29 \%$ of water loss observed in these animals before being sacrificed (Malik and Storey 2009). Animal water loss was calculated by using the following equation: \% Changed $=\left[\left(\mathrm{W}_{\mathrm{i}}-\mathrm{W}_{\mathrm{d}}\right) /\left(\mathrm{W}_{\mathrm{i}} \times \mathrm{BWC}_{\mathrm{i}}\right)\right] \times 100$, where $\mathrm{W}_{\mathrm{i}}$ represents initial body weight, $\mathrm{W}_{\mathrm{d}}$ represents dehydrated body weight, and $\mathrm{BWC}_{\mathrm{i}}$ represents mean body weight of the frogs before experiment. For tissue sampling, animals were pithed with a needle, and tissues were quickly dissected and frozen in liquid nitrogen followed by storage in $-80^{\circ} \mathrm{C}$ freezer until use.

\section{Luminex multiplex protein assay}

Luminex antibody multiplex assay panels were used in this study to profile expression levels of key proteins in the insulin/Akt signaling pathway and glycolysis. The following 
Luminex kits were used in this study: Akt/mTOR Phosphoprotein 11-plex kit (Millipore; 48611MAG), Human Glycolysis Pathway (Millipore; HGPMAG-27K-01) and housekeeping protein GAPDH (glyceraldehyde 3-phosphate dehydrogenase) single-plex (Millipore; 46667MAG). In the Akt/mTOR panel, phosphorylation levels for the following proteins were measured: insulin receptor (IR) at serine-636, insulin-like growth factor-1 receptor (IGF-1R) at tyrosine-1135/1136, insulin receptor substrate-1 (IRS-1) at tyrosine-1162/1163, glycogen synthase kinase-3 beta (GSK-3 $\beta$ ) at serine-9, protein kinase B (PKB/Akt) at serine-473, phosphatase and tensin homolog (PTEN) at serine-380, tuberous sclerosis 2 (TSC2) at serine939, mechanistic target of rapamycin (mTOR) at serine-2448, ribosomal protein S6 kinase (P70S6K) at threonine-412, and ribosomal protein S6 (RPS6) at serine-235/236. In the Human Glycolysis panel, the total levels for the following proteins were measured: enolase 1 (ENO1), glucose-6-phsophate isomerase (G6PI), hypoxia inducible factors-1 alpha (HIF-1 $\alpha$ ), L-lactate dehydrogenase A chain (LDHA), L-lactate dehydrogenase B chain (LDHB), pyruvate kinase isoform M2 (PKM2), and transketolase (TK).

To prepare lysates for the Luminex assay, $~ 50 \mathrm{mg}$ of $X$. laevis brain, heart, and kidney tissues were homogenized in protein lysis buffer with a Dounce homogenizer supplemented with $2 \mathrm{mM}$ of phenylmethylsulfonyl fluoride (PMSF). Protein lysates were extracted by centrifuging the homogenate at $4000 \times \mathrm{g}$ for $10 \mathrm{~min}$, lysates were subsequently normalized to the same concentration with lysis buffer after the Bradford assay. Luminex assays were carried out according to manufacturer's protocol with a Luminex 200 machine, assay protocols were previously described in detail (Biggar et al. 2015). The GAPDH protein levels were also measured with the Luminex 200 machine and used as the housekeeping protein for data normalization. 
Protein sequence alignment

The human amino acid sequences for proteins measured in this study were retrieved from the NCBI gene database, and the protein sequences for $X$. laevis orthologues were retrieved from XenBase (www.xenbase.org/) (Karpinka et al. 2015). Geneious software was used to align the protein sequences flanking the phosphorylation residues measured in this study.

\section{Statistical analysis}

Data collected from the Luminex assays were expressed as relative protein levels by normalizing all values to the first control sample that was arbitrary set to 1 . All numerical data are expressed as means $\pm \operatorname{sem}(n=4$ samples from independent animals $)$; statistical significance was determined by a One-Way ANOVA Tukey's test using the SigmaPlot 12.0 statistical package.

\section{Results}

Key regulators of the insulin/Akt signaling cascade is inhibited during dehydration stress.

To begin characterizing regulation of the insulin/Akt signaling cascade during dehydration stress in $X$. laevis, we used a Luminex antibody array to measure the phosphorylation levels of key proteins within this signaling axis. Amino acid sequence alignments show that all the phosphorylation residues and its proximal flanking sequences for proteins measured in this study were highly conserved between $X$. laevis and human (Figures 13). The insulin signaling pathway is comprised of a phosphorylation cascade, with each phosphorylation modification serving to either activate or inhibit the function of the target 
protein (Figure 1A). In response to growth factors or stimuli, the receptor proteins IR and IGF$1 \mathrm{R}$ is activated through phosphorylation, and subsequently phosphorylates its cellular protein target IRS-1. As shown in Figure 1B, phosphorylation of p-IGF-1R was reduced in the brain and heart of $X$. laevis during medium dehydration by $58 \%$ and $43 \%$ respectively. While the phosphorylation levels for both p-IR and p-IRS-1 showed similar pattern of reduction, it was not statistically significant (Figure 1C-D).

Downstream of the insulin receptors, the Akt kinase act as the hub of this signaling cascade, functioning to phosphorylate many downstream substrates. In all three tissues examined in this study, phosphorylation of Akt was significantly reduced in the brain showing a $47 \%$ and $61 \%$ reduction during medium and high dehydration respectively, the heart showing $43 \%$ and $77 \%$ reduction during medium and high dehydration respectively, and the kidney showing 54\% and 59\% reduction during medium and high dehydration respectively (Figure 2A). The upstream regulation of Akt is partly mediated through the action of the PTEN phosphatase, which act as a negative regulator of the insulin signaling pathway by catalyzing the dephosphorylation of phosphatidylinositol $(3,4,5)$-trisphosphate (PIP3) to phosphatidylinositol $(4,5)$-bisphosphate (PIP2) (Wu et al. 2015); phosphorylation levels of PTEN at its inactivating residue was unchanged during dehydration stress in all three tissues (Figure 2B). Two direct downstream targets of Akt are the TSC2 and GSK-3 $\beta$ proteins; when Akt is active, it inhibits the functions of these two target proteins by phosphorylation. TSC2 is part of the TSC-1/2 inhibitory complex that blocks the activity of the mTOR kinase, phosphorylation levels of TSC2 at its inactivating residue was reduced in the heart during high dehydration by $45 \%$ (Figure $2 \mathrm{C}$ ). Meanwhile, phosphorylated levels of GSK-3 $\beta$ remain unchanged across the tissues examined during 
dehydration stress (Figure 2D); GSK-3 $\beta$ is a serine/threonine kinase that phosphorylates and inhibits the activity of glycogen synthase.

An integral part of the insulin/Akt signaling axis is the mTOR protein, which is a nutrient and energy sensor that controls metabolic homeostasis. Part of how mTOR regulates cellular homeostasis is via its control of protein synthesis by regulating its downstream target which includes P70S6K. When mTOR is active, it phosphorylates and activates P70S6K, which subsequently phosphorylates the ribosomal S6 protein that is part of the $40 \mathrm{~S}$ ribosomal complex. As seen in Figure 3A, the phosphorylation of mTOR at its activation residue was significantly reduced during medium dehydration stress in the brain by $34 \%$ and heart by $37 \%$. However, in both tissues, neither P70S6K nor RPS6 showed changes in its phosphorylation (Figure 3B-C). Interestingly in the kidney, dehydration stress had no effects on the phosphorylation of mTOR, furthermore, the phosphorylation of P70S6K was elevated by 3.3-folds during dehydration, coupled with an increase in phosphorylation of RPS6 by 15.6-folds and 12.2-folds during medium and high dehydration stress respectively (Figure 3B-C). Overall, these results show that key components of the insulin/Akt axis is inhibited in in the brain and the heart of $X$. laevis during dehydration stress, and this pathway may be regulated in a tissue specific manner as many of the inhibitory modifications were not observed in the kidney tissue.

HIF-1 $\alpha$ is selectively overexpressed during dehydration stress, but does not influence expression of proteins in glycolysis

The mTOR kinase as mentioned earlier is part of a nutrient sensing complex that can coordinate metabolic adjustments in response to changes in nutrient and energy level of the cell (Ma and Blenis 2009). One mechanism by which mTOR has been shown to influence energy 
metabolism is by promoting glycolysis via the hypoxic sensor HIF-1 $\alpha$ protein (Semenza et al. 1994, Weichhart et al. 2015). In this pathway, activation of mTOR drives the translation of HIF$1 \alpha$ protein expression (Weichhart et al. 2015), which can subsequently promote glycolysis by directly trans-activating the gene expression of glycolytic enzymes (Kim et al. 2006, Semenza et al. 1994). To determine whether phosphorylation changes observed for the mTOR protein during dehydration stress (Figure 3A) may influence expression of glycolytic proteins via a mechanism that involves HIF-1 $\alpha$, we used a Luminex antibody panel that profiled the human glycolysis pathway. The glycolytic proteins measured in this study were highly conserved between $X$. laevis and human with sequence similarities in the ranges of $78.8 \%-89.4 \%$ (Figure $4 \mathrm{~A}$ ). Since the phosphorylation of mTOR at its activation residue was reduced in the heart and brain during dehydration stress, we might expect a similar reduction in the HIF-1 $\alpha$ protein levels. As shown in Figure 4B, the total protein levels of HIF-1 $\alpha$ remained unchanged in the brain and the kidney during dehydration stress, and HIF-1 $\alpha$ was significantly elevated in the heart during medium dehydration by 2.15-folds (Figure 4B). When we measured the protein levels of six glycolysis related enzymes, we found that none of the protein levels changed in any of the three tissues during dehydration stress (Figure 4C-F). Overall these results show that protein levels of many enzymes within glycolysis are not significantly affected in X. laevis during dehydration stress, and that the expression levels of HIF-1 $\alpha$ proteins may also be regulated independently of mTOR in this context.

\section{Discussion}

Estivation is an adaptive stress response used by $X$. laevis in the wild to survive during periods of drought, and in this study, we used dehydration stress as a model to understand how 
the insulin signaling cascade and glycolysis are regulated during this period of dormancy. We found that during dehydration stress, key components of the insulin signaling pathway (IGF-1R, Akt, and mTOR) show significant reduction in its phosphorylation levels at residues that represent protein activation, and this regulation was observed in a tissue specific manner (Figure 1-3). While animals under estivation experience periods of hypoxia, which is a cellular state that has previously been shown to influence glycolysis via a mechanism that involves mTOR and HIF-1 $\alpha$, we found that the expression of glycolytic proteins examined in this study remained unchanged, suggesting that glycolysis may not be affected at the translational level during dehydration stress. Suppression of the insulin signaling cascade during periods of metabolic stress is not a novel finding, rather, our study suggests that $X$. laevis may inhibit the insulin signaling pathway in a similar manner to those observed during mammalian hibernation, and is used as a strategy to promote metabolic depression during estivation when food and water resources become limited (Abnous et al. 2008, Cai et al. 2004, McMullen and Hallenbeck 2010, Wu and Storey 2012).

\section{Insulin signaling and protein synthesis during dehydration stress}

Inhibition of protein synthesis has been previously documented in both vertebrate and invertebrate models of estivation, protein synthesis was found to be reduced by $67 \%$ in the Australian desert frog Neobatrachus centralis and by $77-82 \%$ in the land snail Otala lactea during estivation (Fuery et al. 1998, Ramnanan et al. 2009). These findings were not unexpected as animals under estivation are also under starvation stress due to reduced food availability, and likely cannot allocate energetic resources to maintain normal protein synthesis which consumes approximately 5 ATP per peptide bonds formed (Storey and Storey 2012). The significant 
decrease in phosphorylation levels of mTOR at its activation residue would suggest that protein synthesis is also likely affected in a similar manner in the brain and heart of X. laevis during dehydration stress. While we did not observe a correlated decrease in the phosphorylation of ribosomal proteins, mTOR is known to exert its control over protein synthesis through regulating a large number of downstream effectors, with many of these proteins directly involved in the translation machinery including 4E-BP1 (eukaryotic translation initiation factor 4E-binding protein 1), eIF4B (eukaryotic translation initiation factor 4B), eIF4G (eukaryotic translation initiation factor 4G), and eEF2 (eukaryotic elongation factor 2) (Hay and Sonenberg 2004).

In hibernating animals, translational arrest is achieved through a similar decrease in mTOR phosphorylation, but this suppression of protein synthesis is also aided by the dramatic decrease in the ambient and core body temperature of the animal (Frerichs et al. 1998, Wu and Storey 2012). In X. laevis, such dramatic shift in ambient temperature do not occur during dehydration stress, however, protein translation may be affected by the relative changes in the osmolarity of the animal during estivation. In mammalian cells, changes in the state of hydration can trigger osmo-sensing pathways that influence metabolism. Exposure to hyperosmolarity in cells can cause cell shrinkage that leads to insulin resistance and trigger catabolic pathways; conversely, swelling of the cells by hypoosmolarity can trigger anabolic pathways that stimulate proliferation (Schliess et al. 2006). In tissues that are sensitive to insulin, dehydration stress can contribute to insulin resistance, with hyperosmotic levels of $\mathrm{NaCl}$ and urea shown to impair insulin induced glucose uptake in human cells (Komjati et al. 1989). Part of how hyperosmolarity inhibits with the insulin signaling pathway is by inactivating the Akt kinase, which can subsequently interfere with and reduce the rate of protein synthesis through its control of the mTOR kinase (Meier et al. 1998). Results from our study which showed reduced levels of 
Akt and mTOR phosphorylation in the heart and brain could suggest that $X$. laevis may take advantage of the inhibitory effects hyperosmolarity has on the insulin signaling cascade, and utilizes this as a potential mechanism to inhibit protein synthesis during estivation. This idea would support osmolyte regulation as an important mechanism to not only assist in water retention of the animal during dehydration, but also as a mean to inhibit protein synthesis during estivation to help conserved energy and establish a hypometabolic state.

\section{Effects of dehydration on the kidney}

The kidney tissues are exposed to significant fluctuations in osmolarity under normal physiological conditions, as such, renal cells are able to tolerate and adapt to a wide range of osmotic conditions (Brocker et al. 2012). During periods of dehydration stress such as those experienced by $X$. laevis, maintenance of kidney functions may be of high importance as levels of nitrogenous osmolytes are significantly increased to promote water retention (Storey and Storey 2012). But such drastic increase in osmolyte levels can cause serious damages to renal cells, as hyperosmotic stress has been shown to result in protein and DNA damages triggered by elevated levels of reactive oxygen species (Zhang et al. 2004). While increased production of nitrogenous osmolyte is an important strategy to defend against over-desiccation of bodily fluids, additional cellular mechanisms may be needed to defend against the intracellular damages caused by hyperosmolarity. A previous study has shown that several members of the mitogenactivated protein kinase (MAPK) signaling cascade are activated in the kidney of $X$. laevis during dehydration stress (Malik and Storey 2009), these include MEKK (MAP kinase kinase kinase), MEK-1 (MAP kinase kinase), ERK-1/2 (extracellular signal regulated kinase), and p90RSK (p90 ribosomal s6 kinase). The MAPK signaling cascade is one of the well-known 
pathways that respond that environmental stress, and are involved in regulating a variety of amphibian stress response that include anoxia, freezing, and osmotic stress (Cowan and Storey 2003). Activation of the p90RSK in the kidney during dehydration stress is intriguing, as it is involved in the regulation of cell growth and proliferation, and when activated, p90RSK can promote gene transcription, protein translation, and cell survival (Anjum and Blenis 2008). Part of how p90RSK stimulates cell growth is through activating protein translation, by directly phosphorylating the ribosomal S6 protein (Schwab et al. 1999, Wettenhall et al. 1992). In this mechanism, p90RSK is able to regulate protein translation via a signaling pathway that is separate from the insulin/Akt axis (Anjum and Blenis 2008). This may partly explain the discrepancy of reduced Akt signaling and increased ribosomal protein activation in the kidney observed in our study (Figure 2-3). Interestingly, p90RSK was found to be significantly inhibited in the X. laevis heart during dehydration stress (Malik and Storey 2009), supporting evidence of tissue specific regulation of cellular processes in $X$. laevis during dehydration stress. While global translation may be reduced during estivation, it can be proposed that the kidney tissue may retain some of its protein synthesis capacity. This is may be achieved by activating ribosomal proteins through the MAPK/p90RSK signaling pathway, which could function as a mechanism to compensate for the negative effects hyperosmolarity has on the insulin signaling axis. Preservation of protein synthesis in the kidney tissue could serve to maintain expression of proteins that may be important for upholding osmotic balance, or in defending against osmotic stress associated protein and DNA damages accrued during prolonged periods of dehydration experienced during estivation.

Hypoxia response and glycolysis during dehydration stress 
During dehydration stress, the amphibian cardiovascular system experience increases in blood viscosity and decreases in blood volume, which can significantly reduce the perfusion of oxygenated blood to peripheral tissues of the animal (Hillman 1987, 1988). As such, amphibian internal organs can become hypoxic during estivation, and this is further exacerbated with animals burrowing into poorly ventilated soil to escape from the hot and arid surface conditions (Storey and Storey 2012). When cells are subject to hypoxic stress, there is a propensity to rely on anaerobic respiration to generate ATP, suggesting that glycolysis may be altered in animals under dehydration stress. Supporting this, a previous study has shown that in the skeletal muscle of $X$. laevis, the activity of hexokinase which catalyzes the first step of glycolysis, was increased by 3.4-folds during dehydration stress (Childers and Storey 2016). Part of how glycolysis can be activated during hypoxia is through the HIF-1 $\alpha$ protein, which can directly trans-activate genes encoding glycolytic enzymes (Kim et al. 2006, Semenza et al. 1994), and recent studies have shown that the mTOR pathway can directly influence this process by mediating translation of the HIF-1 $\alpha$ protein (Weichhart et al. 2015).

In our study, we observed that the HIF-1 $\alpha$ protein was up-regulated by $\sim 2$-folds in the heart during dehydration stress, which would support evidence of hypoxia stress in $X$. laevis during estivation. Up-regulation of HIF-1 $\alpha$ in the heart has been associated with cardioprotection, with HIF-1 $\alpha$ shown to directly activate the transcription of genes that mediate the adaptive hypoxia/ischemia stress response (Cai et al. 2003). Interestingly, this hypoxia response in $X$. laevis does not appear to be linked to changes in the regulation of glycolysis, as protein levels of many glycolytic enzymes were unchanged during dehydration. However, this does not rule out that glycolysis may still be differentially regulated during dehydration stress. It is possible that modifications to glycolytic flux may be controlled by post-translational 
modifications (PTM) that influence the activity of each enzymes rather than affecting its expression, and evidence of PTM via reversible phosphorylation of key glycolytic enzymes has previously been documented in estivation of land snails (Ramnanan and Storey 2006, Whitwam and Storey 1990).

\section{Conclusions}

In this study, we show that the insulin signaling pathway is suppressed in the heart and brain tissues of the $X$. laevis during periods of dehydration stress. While suppression of the insulin signaling pathway has previously been shown in cells during hyperosmotic stress, we suggest that $X$. laevis may take advantage of this response to shut down protein synthesis through the mTOR pathway as a mean to limit energy consumption during estivation. We also observed that in the kidney tissue, proteins involved in ribosome assembly are highly activated during dehydration, and this may be linked to the previous observation of an activated MAPK signaling pathway in this tissue during dehydration stress (Malik and Storey 2009). Maintenance of kidney functions may serve to support the osmoregulation of $X$. laevis which experiences significant increase in levels of nitrogenous osmolytes during dehydration stress. Furthermore, we observed that the heart tissue may experience periods of hypoxia during dehydration stress, as evident by the up-regulation of the HIF-1 $\alpha$ protein; however, this increase in hypoxia response does not appear to be linked to the regulation in the levels of the glycolytic proteins measured in this study. In summary, our study provides new molecular insights into the regulation of the insulin signaling and glycolysis pathway during dehydration stress, and show that many of the proteins within these pathways are regulated in a tissue specific manner, implicating the different 
physiological importance of individual tissues in coordinating the estivation response in $X$. laevis.

\section{Acknowledgements}

We thank J.M. Storey for providing support of the animal preparations and for editorial review of the manuscript.

Funding: This research was funded by a Discovery grant from the Natural Sciences and Engineering Research Council (NSERC) of Canada to KBS (\#6793), a Grant-in-Aid from the Heart and Stroke Foundation of Canada (\#0005874), and NSERC doctoral scholarships to CWW and SNT.

Conflict of Interest: The authors declare that they have no conflict of interest. 


\section{References}

Abnous, K., Dieni, C.A., and Storey, K.B. 2008. Regulation of Akt during hibernation in Richardson's ground squirrels. Biochim Biophys Acta 1780(2): 185-193.

Anjum, R., and Blenis, J. 2008. The RSK family of kinases: emerging roles in cellular signalling. Nat Rev Mol Cell Biol 9(10): 747-758.

Biggar, K.K., Wu, C.W., Tessier, S.N., Zhang, J., Pifferi, F., Perret, M., and Storey, K.B. 2015. Primate Torpor: Regulation of Stress-activated Protein Kinases During Daily Torpor in the Gray Mouse Lemur, Microcebus murinus. Genomics Proteomics Bioinformatics 13(2): 81-90.

Brocker, C., Thompson, D.C., and Vasiliou, V. 2012. The role of hyperosmotic stress in inflammation and disease. Biomol Concepts 3(4): 345-364.

Cai, D., McCarron, R.M., Yu, E.Z., Li, Y., and Hallenbeck, J. 2004. Akt phosphorylation and kinase activity are down-regulated during hibernation in the 13-lined ground squirrel. Brain Res 1014(1-2): 14-21.

Cai, Z., Manalo, D.J., Wei, G., Rodriguez, E.R., Fox-Talbot, K., Lu, H., Zweier, J.L., and Semenza, G.L. 2003. Hearts from rodents exposed to intermittent hypoxia or erythropoietin are protected against ischemia-reperfusion injury. Circulation 108(1): 79-85.

Carracedo, A., and Pandolfi, P.P. 2008. The PTEN-PI3K pathway: of feedbacks and cross-talks. Oncogene 27(41): 5527-5541.

Childers, C.L., and Storey, K.B. 2016. Post-translational Regulation of Hexokinase Function and Protein Stability in the Aestivating Frog Xenopus laevis. Protein J 35(1): 61-71.

Cowan, K.J., and Storey, K.B. 2003. Mitogen-activated protein kinases: new signaling pathways functioning in cellular responses to environmental stress. J Exp Biol 206(Pt 7): 1107-1115.

Frerichs, K.U., Smith, C.B., Brenner, M., DeGracia, D.J., Krause, G.S., Marrone, L., Dever, T.E., and Hallenbeck, J.M. 1998. Suppression of protein synthesis in brain during hibernation involves inhibition of protein initiation and elongation. Proc Natl Acad Sci U S A 95(24): 1451114516.

Fuery, C.J., Withers, P.C., Hobbs, A.A., and Guppy, M. 1998. The role of protein synthesis during metabolic depression in the Australian desert frog Neobatrachus centralis. Comp Biochem Physiol A Mol Integr Physiol 119(2): 469-476.

Hay, N., and Sonenberg, N. 2004. Upstream and downstream of mTOR. Genes Dev 18(16): 1926-1945. 
Hillman, S.S. 1987. Dehydrational Effects on Cardiovascular and Metabolic Capacity in Two Amphibians. Physiol Zool 60(5): 608-613.

Hillman, S.S. 1988. Dehydrational Effects on Brain and Cerebrospinal Fluid Electrolytes in Two Amphibians. Physiol Zool 61(3): 254-259.

Karpinka, J.B., Fortriede, J.D., Burns, K.A., James-Zorn, C., Ponferrada, V.G., Lee, J., Karimi, K., Zorn, A.M., and Vize, P.D. 2015. Xenbase, the Xenopus model organism database; new virtualized system, data types and genomes. Nucleic Acids Res 43(Database issue): D756-763.

Kim, J.W., Tchernyshyov, I., Semenza, G.L., and Dang, C.V. 2006. HIF-1-mediated expression of pyruvate dehydrogenase kinase: a metabolic switch required for cellular adaptation to hypoxia. Cell Metab 3(3): 177-185.

Kimura, K.D., Tissenbaum, H.A., Liu, Y., and Ruvkun, G. 1997. daf-2, an insulin receptor-like gene that regulates longevity and diapause in Caenorhabditis elegans. Science 277(5328): 942946.

Komjati, M., Kastner, G., Waldhäusl, W., and Bratusch-Marrain, P. 1989. Effect of hyperosmolality on basal and hormone-stimulated hepatic glucose metabolism in vitro. Eur J Clin Invest 19(2): 128-134.

Krivoruchko, A., and Storey, K.B. 2010. Forever young: mechanisms of natural anoxia tolerance and potential links to longevity. Oxid Med Cell Longev 3(3): 186-198.

Laplante, M., and Sabatini, D.M. 2009. mTOR signaling at a glance. J Cell Sci 122(Pt 20): 35893594.

Loveridge, J.P., and Withers, P.C. 1981. Metabolism and Water Balance of Active and Cocooned African Bullfrogs Pyxicephalus adspersus. Physiol Zool 54(2): 203-214.

Ma, X.M., and Blenis, J. 2009. Molecular mechanisms of mTOR-mediated translational control. Nat Rev Mol Cell Biol 10(5): 307-318.

Malik, A.I., and Storey, K.B. 2009. Activation of extracellular signal-regulated kinases during dehydration in the African clawed frog, Xenopus laevis. J Exp Biol 212(Pt 16): 2595-2603.

Manning, B.D., and Cantley, L.C. 2007. AKT/PKB signaling: navigating downstream. Cell 129(7): 1261-1274.

McMullen, D.C., and Hallenbeck, J.M. 2010. Regulation of Akt during torpor in the hibernating ground squirrel, Ictidomys tridecemlineatus. J Comp Physiol B 180(6): 927-934. 
Meier, R., Thelen, M., and Hemmings, B.A. 1998. Inactivation and dephosphorylation of protein kinase Balpha (PKBalpha) promoted by hyperosmotic stress. EMBO J 17(24): 7294-7303.

Merkle, S., and Hanke, W. 1988. Long-term starvation in Xenopus laevis Daudin--II. Effects on several organs. Comp Biochem Physiol A Comp Physiol 90(3): 491-495.

Peña-Villalobos, I., Narváez, C., and Sabat, P. 2016. Metabolic cost of osmoregulation in a hypertonic environment in the invasive African clawed frog Xenopus laevis. Biol Open 5(7): 955-961.

Ramnanan, C.J., Allan, M.E., Groom, A.G., and Storey, K.B. 2009. Regulation of global protein translation and protein degradation in aerobic dormancy. Mol Cell Biochem 323(1-2): 9-20.

Ramnanan, C.J., and Storey, K.B. 2006. Glucose-6-phosphate dehydrogenase regulation during hypometabolism. Biochem Biophys Res Commun 339(1): 7-16.

Romspert, A.P. 1976. Osmoregulation of the african clawed frog. Xenopus laevis, in hypersaline media. Comp Biochem Physiol A Mol Integr Physiol 54(2): 207-210.

Schliess, F., Richter, L., vom Dahl, S., and Häussinger, D. 2006. Cell hydration and mTORdependent signalling. Acta Physiol (Oxf) 187(1-2): 223-229.

Schwab, M.S., Kim, S.H., Terada, N., Edfjäll, C., Kozma, S.C., Thomas, G., and Maller, J.L. 1999. p70(S6K) controls selective mRNA translation during oocyte maturation and early embryogenesis in Xenopus laevis. Mol Cell Biol 19(4): 2485-2494.

Semenza, G.L., Roth, P.H., Fang, H.M., and Wang, G.L. 1994. Transcriptional regulation of genes encoding glycolytic enzymes by hypoxia-inducible factor 1. J Biol Chem 269(38): 2375723763.

Seymour, R.S. 1973. Energy Metabolism of Dormant Spadefoot Toads (Scaphiopus). Copeia 1973(3): 435-445.

Storey, K.B. 2010. Out cold: biochemical regulation of mammalian hibernation - a mini-review. Gerontology 56(2): 220-230.

Storey, K.B., and Storey, J.M. 2012. Aestivation: signaling and hypometabolism. J Exp Biol 215(Pt 9): 1425-1433.

Storey, K.B., and Storey, J.M. 2017. Molecular Physiology of Freeze Tolerance in Vertebrates. Physiol Rev 97(2): 623-665. 
Taniguchi, C.M., Emanuelli, B., and Kahn, C.R. 2006. Critical nodes in signalling pathways: insights into insulin action. Nat Rev Mol Cell Biol 7(2): 85-96.

Weichhart, T., Hengstschläger, M., and Linke, M. 2015. Regulation of innate immune cell function by mTOR. Nat Rev Immunol 15(10): 599-614.

Wettenhall, R.E., Erikson, E., and Maller, J.L. 1992. Ordered multisite phosphorylation of Xenopus ribosomal protein S6 by S6 kinase II. J Biol Chem 267(13): 9021-9027.

Whitwam, R.E., and Storey, K.B. 1990. Pyruvate Kinase from the Land Snail Otala lactea: Regulation by Reversible Phosphorylation During Estivation and Anoxia. J Exp Biol 154(321337).

Wu, C.W., Bell, R.A., and Storey, K.B. 2015. Post-translational regulation of PTEN catalytic function and protein stability in the hibernating 13-lined ground squirrel. Biochim Biophys Acta 1850(11): 2196-2202.

Wu, C.W., Biggar, K.K., and Storey, K.B. 2013. Dehydration mediated microRNA response in the African clawed frog Xenopus laevis. Gene 529(2): 269-275.

Wu, C.W., and Storey, K.B. 2012. Regulation of the mTOR signaling network in hibernating thirteen-lined ground squirrels. J Exp Biol 215(Pt 10): 1720-1727.

Wu, C.W., and Storey, K.B. 2016. Life in the cold: links between mammalian hibernation and longevity. Biomol Concepts 7(1): 41-52.

Zhang, J., and Storey, K.B. 2013. Akt signaling and freezing survival in the wood frog, Rana sylvatica. Biochim Biophys Acta 1830(10): 4828-4837.

Zhang, Z., Dmitrieva, N.I., Park, J.H., Levine, R.L., and Burg, M.B. 2004. High urea and NaCl carbonylate proteins in renal cells in culture and in vivo, and high urea causes 8-oxoguanine lesions in their DNA. Proc Natl Acad Sci U S A 101(25): 9491-9496. 


\section{Figure legends}

Figure 1. Insulin receptor and substrate control in X. laevis during dehydration. A) Graphical illustration of the proteins in the insulin/Akt signaling cascade measured in this study; protein levels of PI3K and PDK-1 were not measured in this study. Relative phosphorylation levels of B) p-IGF-1R (Y1135/1136), C) p-IR (Y1162/1163), and D) p-IRS-1 (S636) in the brain, heart and kidney of $X$. laevis during dehydration stress. Shown next to the histograms are the conserved sites of phosphorylation residue between human and $X$. laevis measured in this study, along with the effects of phosphorylation on protein function. ${ }^{*} \mathrm{P}<0.05$ compared to corresponding control as determined by One-way ANOVA with $\mathrm{N}=3-4$ samples.

Figure 2. Akt kinase is inactivated during dehydration stress. Relative phosphorylation levels of A) p-AKT (S473), B) p-PTEN (S380), C) p-TSC2 (S939), and D) p-GSK-3ß (S9) in the brain, heart, and kidney of $X$. laevis during dehydration stress. Shown next to the histograms are the conserved sites of phosphorylation residue between human and $X$. laevis measured in this study, along with the effects of phosphorylation on protein function. $* \mathrm{P}<0.05, * * \mathrm{P}<0.01$, and $* * * \mathrm{P}<0.001$ compared to corresponding control as determined by One-way ANOVA with N=3-4 samples.

Figure 3. mTOR kinase is differentially controlled between tissues during dehydration. Relative phosphorylation levels of A) p-mTOR (S2448), B) p-P70S6K (T412), and p-RPS6 (S235/S236) in the brain, heart, and kidney of $X$. laevis during dehydration stress. Shown next to the histograms are the conserved sites of phosphorylation residue between human and $X$. laevis 
measured in this study, along with the effects of phosphorylation on protein function. $* \mathrm{P}<0.05$ compared to corresponding control as determined by One-way ANOVA with N=3-4 samples.

Figure 4. Levels of many glycolytic proteins are unaffected during dehydration stress. A) Glycolysis related proteins measured in this study along with its total protein conservation percentage between human and X. laevis. B) Relative levels of HIF-1 $\alpha$ protein in the brain, heart, and kidney of $X$. laevis during dehydration. ${ }^{* *} \mathrm{P}<0.01$ compared to corresponding control as determined by One-way ANOVA with N=3-4 samples. Relative protein levels of ENO1, G6PI, LDHA, LDHB, PKM2, and TKT in the C) Brain, D) Heart, and E) Kidney of X. laevis during dehydration stress. F) Graphical illustration of the glycolysis pathway and the target proteins measured in this study. 
A) Growth Factors, Hormones
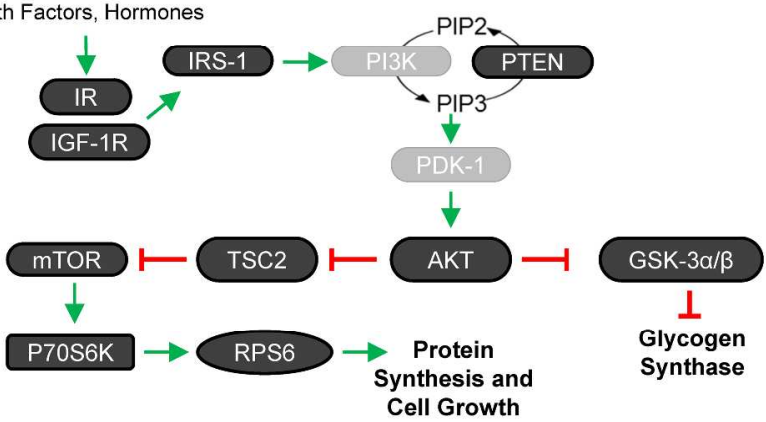

B) $\square$ Control $\square$ Medium Dehydration $\square$ High Dehydration
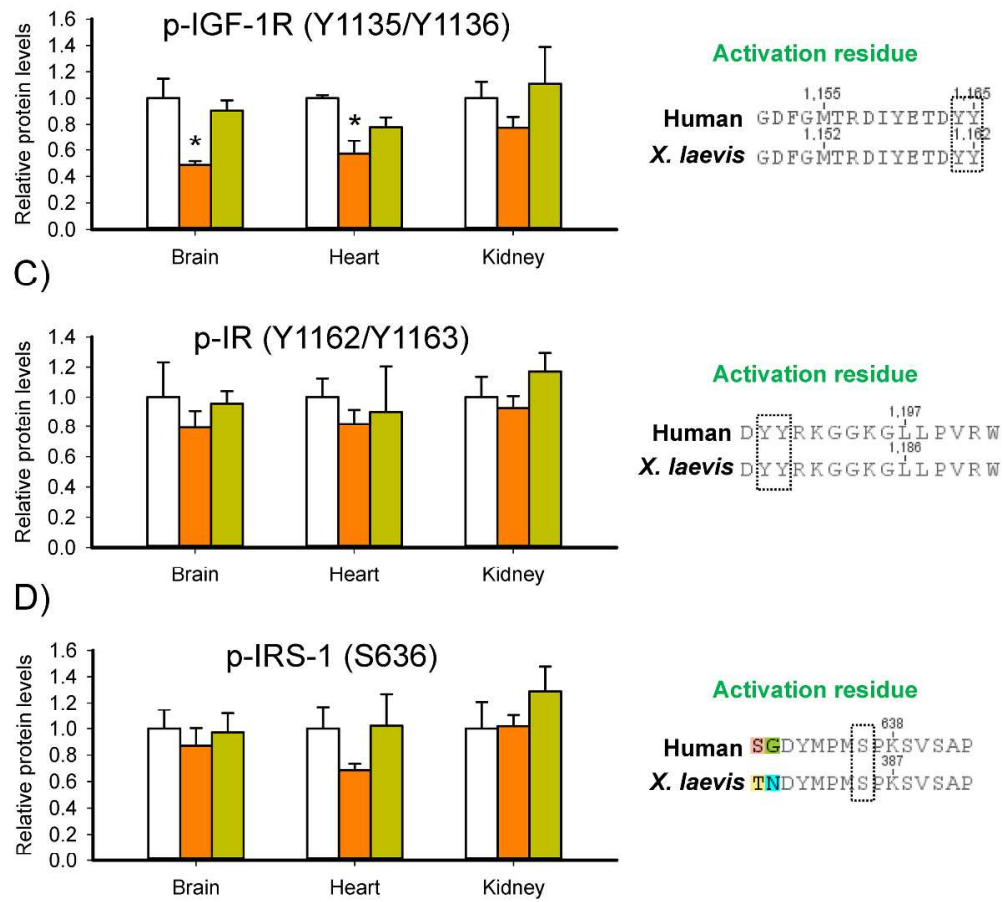

Activation residue

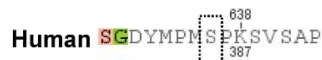

$\boldsymbol{X}$. laevis TNDYMPIS ILSVAP

Figure 1

$279 \times 361 \mathrm{~mm}(300 \times 300 \mathrm{DPI})$ 

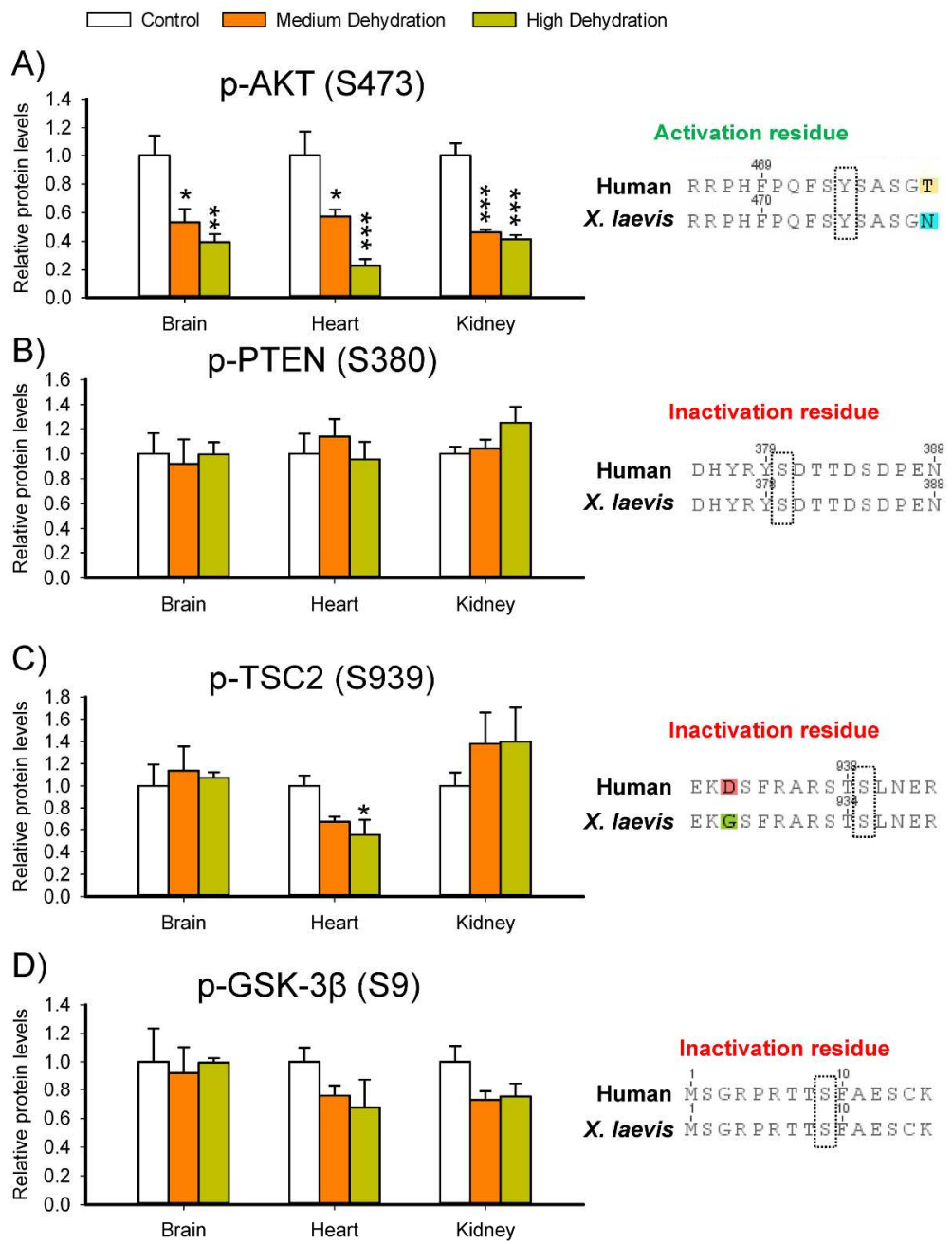

Figure 2

$279 \times 361 \mathrm{~mm}(300 \times 300$ DPI $)$ 

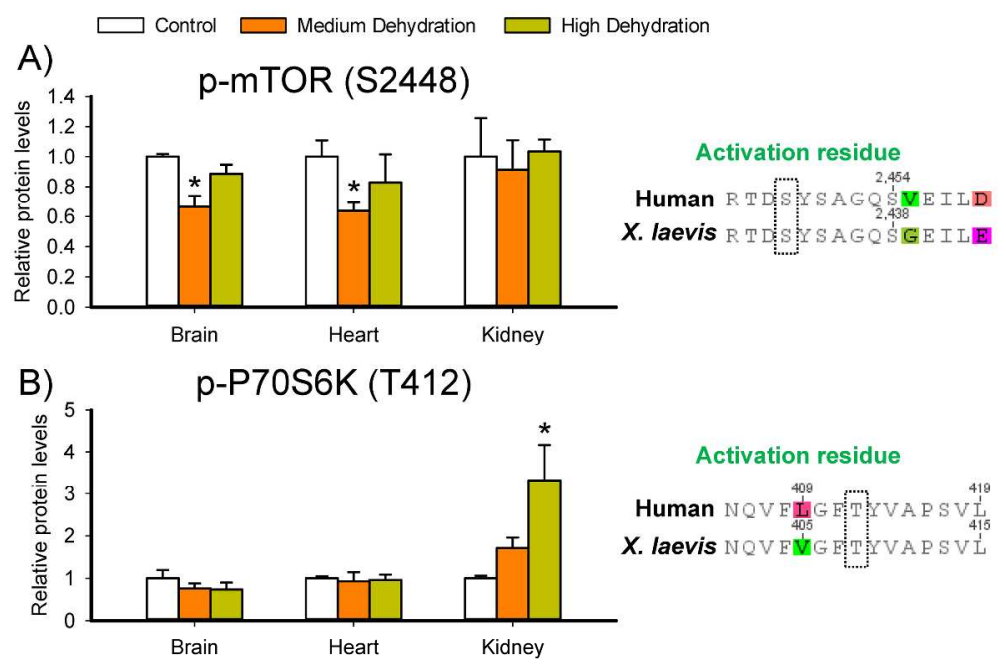

C) p-RPS6 (S235/S236)

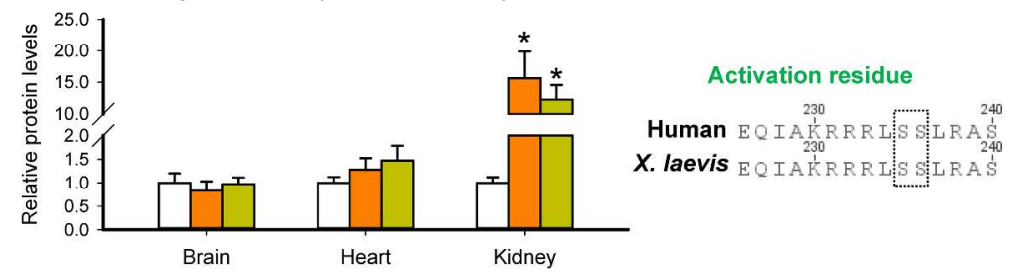

Figure 3

$279 \times 361 \mathrm{~mm}(300 \times 300$ DPI) 


\begin{tabular}{|c|c|}
\hline Glycolysis Protein & $\%$ Protein Conservation \\
\hline Enolase 1 (ENO1) & $87.8 \%$ \\
\hline Glucose-6-phosphate isomerase (G6PI) & $79.2 \%$ \\
\hline L-lactate dehydrogenase A chain (LDHA) & $83.1 \%$ \\
\hline L-lactate dehydrogenase B chain (LDHB) & $82 \%$ \\
\hline Pyruvate kinase isoform M2 (PKM2) & $89.4 \%$ \\
\hline Transketolase (TKT) & $78.8 \%$ \\
\hline
\end{tabular}

B) $\square$ Control $\square$ Medium Dehydration $\square$ High Dehydration
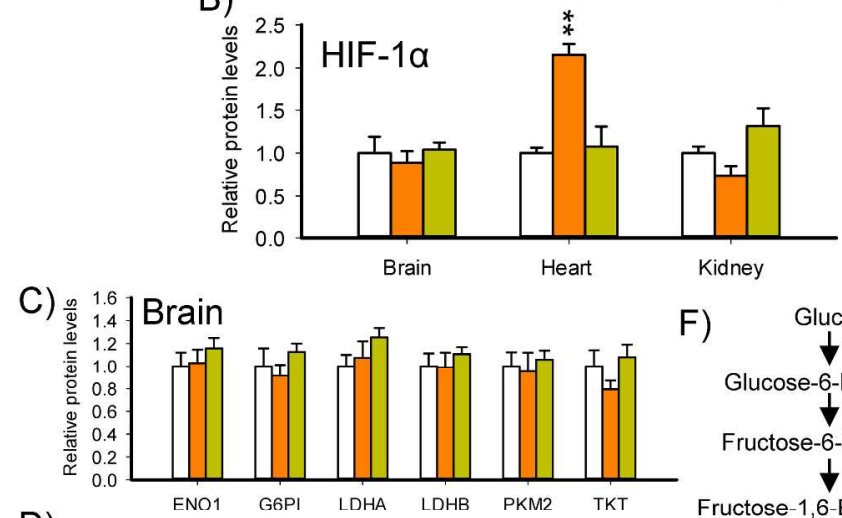

F) Glucose

D) 1.61 1.6

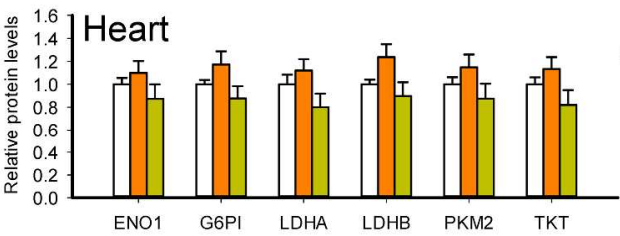

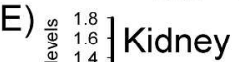
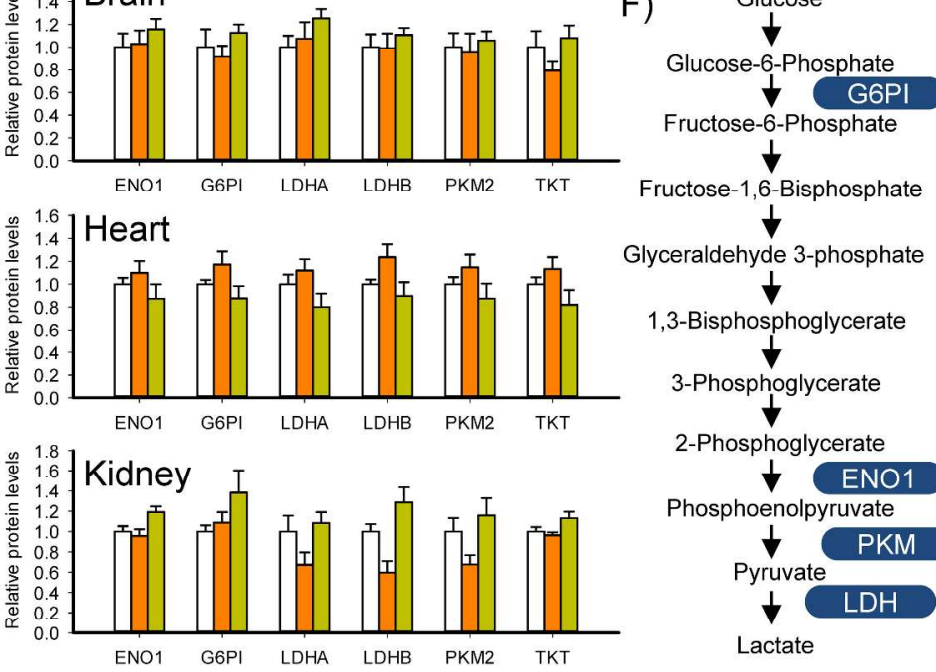

Fructose 1,6 Bisphosphate

Glyceraldehyde 3-phosphate

1,3-Bisphosphoglycerate

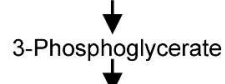

2-Phosphoglycerate

$\downarrow$ ENO1

Phosphoenolpyruvate

$\downarrow$ PKM

Pyruvate

$\downarrow \mathrm{LDH}$

Lactate

Figure 4

$279 \times 361 \mathrm{~mm}(300 \times 300$ DPI $)$ 